\title{
NOUVELLE
}

\section{La kinase GCN2 régule le comportement alimentaire des omnivores afin de maintenir l'homéostasie des acides aminés}

Anne-Catherine Maurin, Céline Jousse, Michelle Balage, Julien Averous, Laurent Parry, Alain Bruhat, Yoan Cherasse, Huiqing Zeng, Yuhong Zhang, Heather Harding, David Ron, Pierre Fafournoux
A.C. Maurin, C. Jousse, M. Balage, J. Averous, L. Parry, A. Bruhat, Y. Cherasse, P. Fafournoux : Unité de Nutrition et métabolisme protéique, Institut National de la Recherche Agronomique (Inra) de Theix, 63122 Saint Genès-Champanelle, France.

fpierre@clermont.inra.fr

H. Zeng, Y. Zhang, H. Harding, D. Ron : Skirball Institute of Biomolecular Medicine, New York University School of Medicine, New York 10016, દ́tats-Unis.
$>$ Dans la nature, le comportement des animaux est dicté en partie par la recherche d'aliments susceptibles d'apporter les nutriments essentiels au fonctionnement de leur organisme. Cette sélection de la nourriture est particulièrement remarquable chez les omnivores, qui ont la capacité d'utiliser des sources diverses pour maintenir un statut nutritionnel équilibré. Outre des facteurs culturels et d'apprentissage, des facteurs innés interviennent également dans la sélection de la nourriture. Par exemple, lorsqu'ils sont soumis à un régime alimentaire unique dépourvu d'un acide aminé indispensable (non synthétisé par l'organisme dans des conditions physiologiques normales), les omnivores sont capables de détecter cette carence et de développer une aversion alimentaire qui consiste à rejeter ce régime considéré comme nocif. Au cours de l'évolution, la capacité de refuser un aliment déséquilibré en acides aminés procure un avantage sélectif à l'animal en lui permettant de privilégier une nourriture équilibrée et mieux adaptée à ses besoins. Cette composante innée implique des mécanismes de détection des déséquilibres alimentaires en acides aminés permettant de déclencher la réponse comportementale.

Des travaux anciens ont impliqué le cerveau, et plus particulièrement une zone appelée cortex piriforme antérieur (APC), dans la mise en place de l'aversion visà-vis de régimes déséquilibrés en aci-

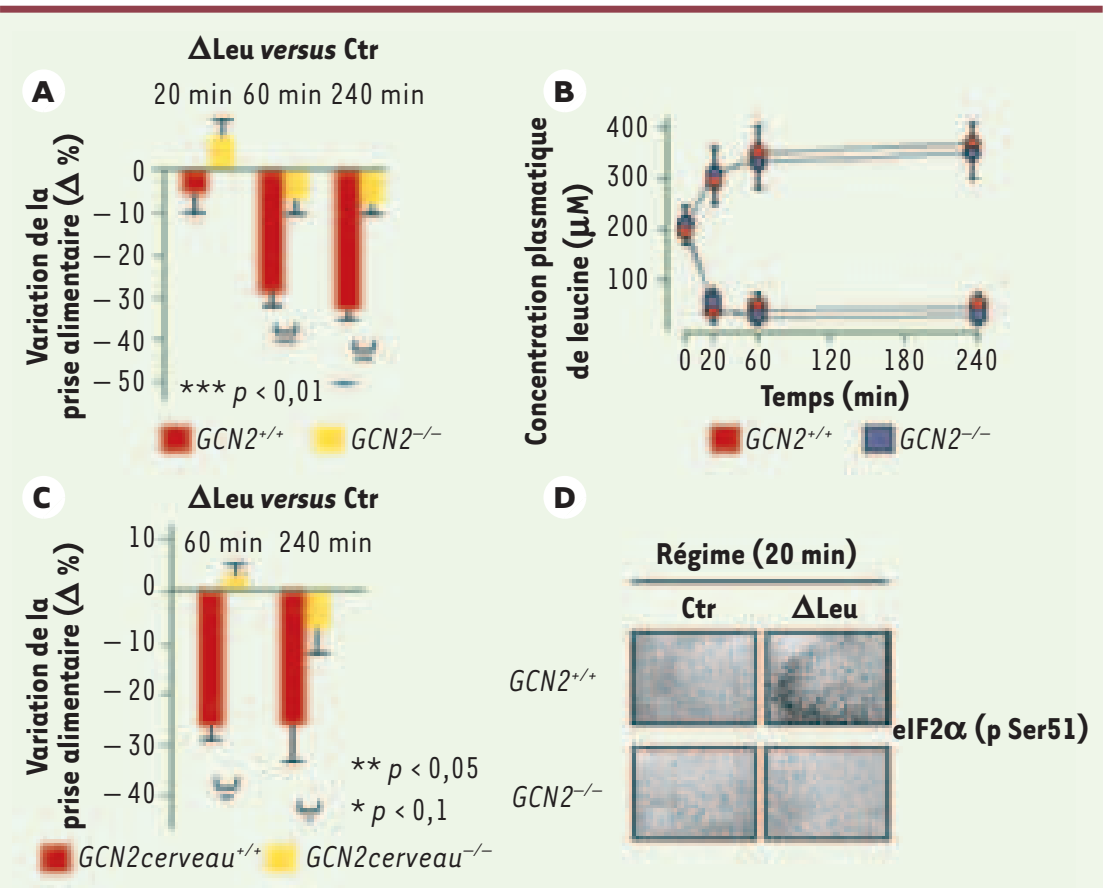

Figure 1. L'aversion alimentaire induite par un déséquilibre nutritionnel en acides aminés implique l'activation de GCN2 dans le cerveau, au niveau du cortex piriforme antérieur. A. L'inactivation de GCN2 supprime l'aversion induite par un déséquilibre nutritionnel en acides aminés. Pourcentage de variation $(\Delta \%)$ de la consommation alimentaire entre un régime carencé en leucine $(\Delta \mathrm{Leu})$ et un régime complet ( $\mathrm{Ctr}$ ) pendant différents intervalles de temps, par des souris sauvages $\left(\mathrm{GCN}^{+/+}\right)$ou invalidées pour GCN2 $\left(G C N 2^{-/-}\right)$. B. Évolution de la concentration plasmatique de leucine après consommation d'un régime complet (Ctr) ou carencé en leucine ( $\triangle$ Leu). C. L'inactivation de GCN2 spécifiquement dans le cerveau annule l'aversion induite par un déséquilibre nutritionnel en acides aminés. $D$. L'inactivation de GCN2 supprime la phosphorylation de elF2 $\alpha$ induite par la carence nutritionnelle en acides aminés dans le cortex piriforme antérieur. Le niveau de phosphorylation de elF2 $\alpha$ a été analysé par immunohistochimie (anticorps anti-elF2 $\alpha[p \operatorname{ser} 51]$ ) sur des coupes de cerveau de souris sauvages $\left(\mathrm{GCN}^{+/+}\right)$ou invalidées pour GCN2 $\left(\mathrm{GCN2}^{-/-}\right)$ayant consommé un régime complet (Ctr) ou carencé en leucine $(\Delta$ Leu $)$ pendant 20 minutes. 
des aminés [1, 2]. Cette zone serait capable de détecter la forte diminution de la concentration sanguine en acides aminés consécutive à la consommation d'un tel régime. Jusqu'à récemment, le mécanisme moléculaire impliqué dans ce processus n'était pas connu. Des travaux récents ont identifié la protéine kinase GCN2 comme le senseur du déficit en acides aminés $[3,4]$.

\section{Les acides aminés en tant} que molécules «signal»: la voie de signalisation

\section{de la protéine kinase GCN2}

Par rapport aux autres macro-nutriments, les acides aminés présentent deux caractéristiques qui rendent leur homéostasie particulièrement dépendante des apports nutritionnels. D'une part, neuf des vingt acides aminés utilisés pour la synthèse protéique sont indispensables, c'est-à-dire que, dans des conditions physiologiques normales, l'organisme d'un adulte en bonne santé n'est pas capable de les synthétiser. D'autre part, il n'existe pas de système spécialisé pour le stockage des acides aminés (équivalent de celui du glycogène dans le foie ou des triglycérides du tissu adipeux). De ce fait, l'ingestion régulière d'un apport protéique équilibré en acides aminés est indispensable. Dans le cas d'une insuffisance d'apport nutritionnel pour un ou plusieurs $\operatorname{acide}(\mathrm{s})$ aminé(s) indispensable(s),

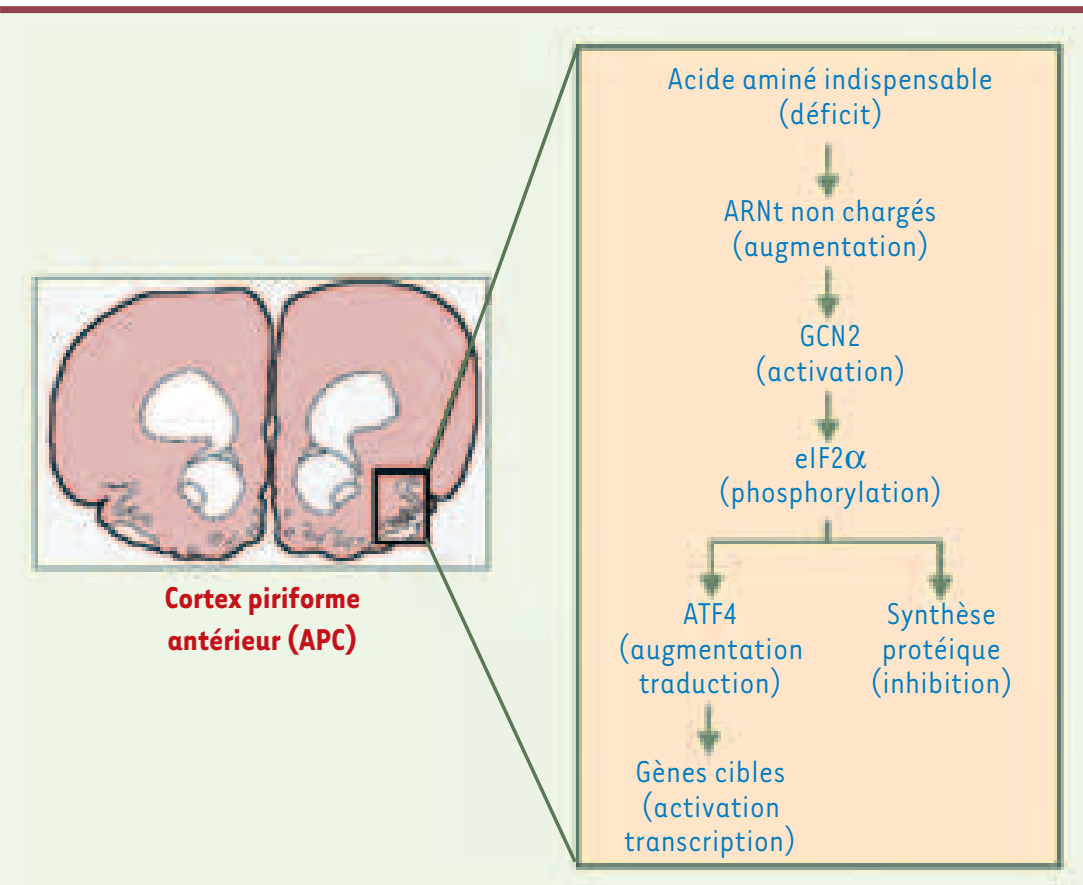

Figure 2. La voie de signalisation GCN2/ATF4 initie la réponse aversive à un déséquilibre nutritionnel en acides aminés dans les neurones de l'APC. Très rapidement, au niveau plasmatique puis au niveau du cerveau ( $A P C)$, la consommation d'un régime déséquilibré se traduit par une forte diminution de la concentration de l'acide aminé indispensable manquant. Dans les neurones de I'APC, la protéine kinase GCN2 est activée par l'augmentation de la concentration des ARNt non chargés résultant du déficit en acides aminés. GCN2 phosphoryle alors la sous-unité $\alpha$ du facteur d'initiation de la traduction elF2, ce qui va avoir deux conséquences : (1) une inhibition de la synthèse protéique, et (2) une augmentation de la traduction du facteur de transcription ATF4. L'induction d'ATF4 est due à une structure particulière de la région 5' non traduite de son ARN messager. Elle conduit à l'activation transcriptionnelle de gènes cibles possédant un élément de réponse aux acides aminés (amino acid response element, AARE).

des mécanismes adaptatifs physiologiques seront mis en route. Ils vont impliquer la modification de l'expression de gènes spécifiques.

Plusieurs voies de signalisation permettant aux organismes de détecter des variations de concentration en acides aminés ont d'abord été décrites chez la levure. Par la suite, des voies homologues ont été retrouvées chez les mammifères. Nos laboratoires ont été particulièrement impliqués dans l'identification de la voie de signalisation passant par la protéine kinase GCN2 et le facteur de transcription ATF4 [5-7]. L'activité enzymatique de GCN2 est inductible par une carence en acides aminés indispensables. En effet, GCN2 possède un domaine homologue de I'histidyl-ARNt synthétase lui permettant de se lier aux ARN de transfert non chargés [8]. II résulte de cette liaison un changement conformationnel permissif pour l'activité kinase. Le seul substrat de GCN2 connu à l'heure actuelle est la sous-unité $\alpha$ du facteur d'initiation de la traduction elF2. La phosphorylation de elF2 $\alpha$ va avoir pour conséquence une inhibition de la formation du complexe d'initiation de la traduction entraînant une forte inhibition de la synthèse protéique. $\varepsilon$ n parallèle, grâce à une structure particulière de sa région 5' non-traduite, la traduction du messager du facteur de transcription ATF4 est augmentée [9, 10]. ATF4 peut alors induire la transcription de gènes cibles possédant dans leur promoteur des éléments de réponse aux acides aminés (amino acid response element, AARE).

\section{Le développement de l'aversion} implique l'activation de la kinase GCN2 dans le cerveau

Nous avons émis I'hypothèse selon laquelle la protéine kinase GCN2 pourrait être impliquée dans le mécanisme de détection de la carence en acides aminés conduisant à l'aversion. Pour cela, nous avons utilisé des souris présentant une invalidation totale du gène codant 
GCN2 (souris GCN2 $2^{-/-}$). Conformément aux données de la littérature décrites chez le rat, nous avons montré que la prise alimentaire de souris sauvages était fortement diminuée en réponse à une carence nutritionnelle en un acide aminé indispensable, la leucine [3]. En revanche, les souris $\mathrm{GCN}^{-/-}$consomment indifféremment les régimes complet ou carencé (Figure 1A), bien que la leucinémie soit fortement diminuée, quel que soit le génotype, par la consommation du régime carencé en leucine (Figure IB). De plus, des souris présentant une invalidation du gène codant GCN2 uniquement dans le cerveau se comportent comme les souris $\mathrm{GCN}^{-/-}$(Figure IC). Afin de localiser le site d'activation de la kinase GCN2 dans le cerveau, nous avons analysé le niveau de phosphorylation de son substrat (elF2 $\alpha$ ) par immuno-histochimie. Vingt minutes de consommation d'un régime carencé en leucine entrânent une forte induction de la phosphorylation de elf2 $\alpha$ dans les neurones de I'APC des souris sauvages, alors qu'aucun marquage n'est obtenu avec les souris $\mathrm{GCN}^{-/-}$(Figure ID).
Nos résultats montrent que l'activité de GCN2 dans le cerveau est indispensable au développement de l'aversion alimentaire résultant du déficit en acides aminés. En couplant la carence en acide aminé à la phosphorylation de elF2 $\alpha$ dans les neurones de I'APC, GCN2 doit agir via ses effets sur la traduction des protéines et/ou la transcription de gènes cibles (Figure 2). Les mécanismes mis en route en aval de l'activation de cette kinase restent à déterminer. Cependant, il est probable que d'autres régions du cerveau soient secondairement impliquées pour aboutir à la réponse comportementale.

La voie GCN2/elF2 $\alpha$ a été conservée chez tous les eucaryotes, de la levure aux mammifères. Chez la levure, cette voie de signalisation régule la biosynthèse des acides aminés en fonction de leur disponibilité. Ces travaux montrent que, chez les omnivores, elle est utilisée pour limiter la consommation d'une nourriture carencée en acides aminés, favorisant par défaut une alimentation équilibrée. $\diamond$ $\mathrm{GCN} 2$ regulates feeding behavior to maintain amino acid homeostasis in omnivores

\section{RÉFÉRENCES}

1. Leung PM, Rogers QR. Importance of prepyriform cortex in food-intake response of rats to amino acids. Am J Physiol 1971; 221 : 929-35.

2. Gietzen, DW. Neural mechanisms in the responses to amino acid deficiency. J Nutr $1993 ; 123: 610-25$.

3. Maurin AC, Jousse C, Averous J, et al. The GCN2 kinase biases feeding behavior to maintain amino acid homeostasis in omnivores. Cell Metab $2005 ; 1$ : 273-7.

4. Hao S, Sharp JW, Ross-Inta CM, et al. Uncharged tRNA and sensing of amino acid deficiency in mammalian piriform cortex. Science 2005 ; 307 : 1776-8.

5. Averous J, Bruhat A, Jousse C, et al. Induction of $\mathrm{CHOP}$ expression by amino acid limitation requires both ATF4 expression and ATF2 phosphorylation. J Biol Chem 2004 ; 279 : 5288-97.

6. Bruhat A, Jousse C, Carraro V, et al. Amino acids control mammalian gene transcription: activating transcription factor 2 is essential for the amino acid responsiveness of the CHOP promoter. Mol Cell Biol $2000 ; 20: 7192-204$.

7. Harding HP, Novoa I, Zhang $Y$, et al. Regulated translation initiation controls stress-induced gene expression in mammalian cells. Mol Cell 2000 ; 6 : 1099-108.

8. Dong J, Qiu H, Garcia-Barrio M, et al. Uncharged tRNA activates GCN2 by displacing the protein kinase moiety from a bipartite tRNA-binding domain. Mol Cell $2000 ; 6: 269-79$.

9. Lu PD, Harding HP, Ron D. Translation reinitiation at alternative open reading frames regulates gene expression in an integrated stress response. J Cell Biol 2004 ; $167: 27-33$.

10. Vattem KM, Wek RC. Reinitiation involving upstream ORFs regulates ATF4 mRNA translation in mammalian cells. Proc Natl Acad Sci USA 2004 ; 101 : 11269-74.

\section{NOUVELLE}

\section{Origine du muscle squelettique : rôles de Pax3/Pax7}

Mounia Lagha, Didier Rocancourt, Frédéric Relaix
Département de Biologie
du développement,
Génétique moléculaire
du développement, CNRS
URA 2578, Institut Pasteur, 25 , rue du Docteur Roux, 75724 Paris Cedex 15, France. frelaix@pasteur.fr essentiellement formé de cellules post-mitotiques: myoblastes en cours de différenciation et fibres musculaires différenciées.

La formation des muscles au cours du développement desvertébrés estun processus progressif avec la mise et/ou Mrf4) apparaissent aux bordures de ce dermomyotome. Elles migrent ensuite entre le dermomyotome et le sclérotome pour former le myotome primaire, premier muscle squelettique du corps $[2,3]$, en place de plusieurs vagues successives de cellules musculaires (dites embryonnaires, fœtales et adultes) dont l'origine cellulaire était restée obscure. Nous avons maintenant identifié une population de myogéniques Myf5, MyoD

sées par l'expression des myogéniques (caractériet des membres dérive des somites, structures embryonnaires segmentées lette axial, et d'un dermomyotome épithélial dorsal à l'origine du derme (peau) et des muscles. Les premières cellules

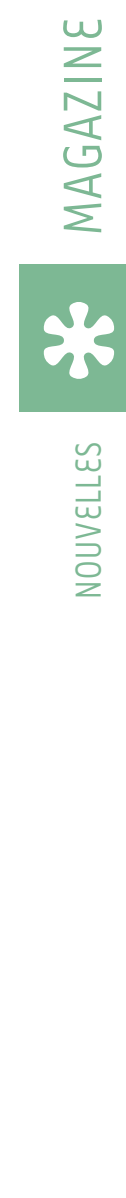

